In Cres. Vol. $5 N^{\circ} 1:$ pp. 59-69, 2014

\title{
DESARROLLO DEL PENSAMIENTO DE LA ESTUDIANTE DE ENFERMERÍA DE LA UNIVERSIDAD NACIONAL DE TRUJILLO*
}

\author{
DEVELOPMENT OF THINKING ON NURSING STUDENTS \\ AT UNIVERSIDAD NACIONAL DE TRUJILLO
}

\author{
Delly Sagastegui Lescano ${ }^{1}$, \\ Carlos Tello Pompa ${ }^{2}$
}

\begin{abstract}
RESUMEN
La presente investigación se llevó a cabo en la Facultad de Enfermería de la Universidad Nacional de Trujillo. Ante la inquietud que surge por la situación de la educación superior en el Perú ${ }^{1}$, como docente de la Facultad de Enfermería, me pregunté: ¿Se desarrolla el pensamiento crítico en la estudiante de Enfermería? Para lo cual los objetivos planteados fueron: identificar, analizar y categorizar el desarrollo del pensamiento crítico en la estudiante de Enfermería. Es una investigación cualitativa, método dialéctico, que busca la explicación de las contradicciones y conflictos en relación con el objeto de estudio. ${ }^{2}$ El procedimiento refleja el análisis y los resultados elaborados según lógica metafísica que entrelazan de manera ecléctica e inconexa tendencias filosóficas. ${ }^{3}$ Los participantes fueron nueve estudiantes, cuatro de V ciclo y cinco de VII ciclo, que aceptaron participar en forma voluntaria. La recolección de la información se realizó mediante la entrevista individual, grabando los discursos, procesándolos inmediatamente identificando, analizando y categorizando la información recabada en forma individual primero y luego agrupando. Se tuvo en cuenta la rigurosidad mediante la confirmabilidad y la triangulación en el proceso; además se tuvo en cuenta las consideraciones éticas de anonimato y confidencialidad. ${ }^{4}$ Las conclusiones finales dieron dos categorías: primaria y secundaria. En la categoría primaria, dicotómica, se desarrolla el pensamiento crítico poco a poco con apoyo de las docentes; no se desarrolla por falta de motivación, confianza, temor, miedo y en proceso de desarrollo exigiéndose más. La categoría secundaria es importante para el cuidado del ser humano.
\end{abstract}

PALABRAS CLAVE: Desarrollo, pensamiento crítico, estudiante de enfermería.

* Recibido: 28 de enero del 2014; aprobado: 7 de junio del 2014.

1 Master. Docente del Departamento de Enfermería. Salud del Adulto y Anciano. Facultad de Enfermería. Universidad Nacional de Trujillo. Trujillo - Perú.

2 Doctor. Docente del Departamento de Enfermería. Salud Familiar y Comunitaria. Facultad de Enfermería. Universidad Nacional de Trujillo. Trujillo - Perú. 


\begin{abstract}
This research was conducted at the Nursing Faculty at the National University of Trujillo, in the concern that arises by state of higher education in Peru ${ }^{1}$ as a professor at the Nursing School I ask me : How do you develop critical thinking in nursing student? To which the objectives were to identify, analyze and categorize, how Critical Thinking develops the nursing student. It is a qualitative research, dialectical method, which tries to explain the contradictions and conflicts in society related to the object of study. ${ }^{2}$ The procedure reflects analysis and results compiled according metaphysical logic of interlocking philosophical eclectic and disjointed manner trends. ${ }^{3}$ Participants were 9 students, 4 of cycle V and 5 of cycle VII, who agreed to participate voluntarily. The data collection was conducted through individual interviews, discourses recording; processing immediately identifying, analyzing and categorizing the information collected individually first and then gathering. Thoroughness by Confirmability and triangulation in the process, and ethical considerations of anonymity and confidentiality ${ }^{4}$ was taken into account. The final conclusions given two categories: primary - secondary, primary category: Dummy: critical slowly with the support of the teachers thought develops, is not developed due to lack of motivation confidence - fear - fear and development process by demanding more. The secondary category is important to the care of the human being.
\end{abstract}

KEY WORDS: Development, nursing student', critical thinking.

\title{
I. INTRODUCCIÓN
}

Estadísticas recientes revelan que el nivel educativo de la población adulta continúa aumentando en el Perú, debido principalmente a que existe un mayor número de jóvenes que completa la secundaria y la educación superior. La matrícula en educación superior se acerca al millón de estudiantes, habiendo aumentado en $75 \%$ respecto de 1990. En el año 2007 existía 3.49 estudiantes matriculados en educación superior por cada 100 habitantes, casi uno por ciento más de los que había en 1990.

La Facultad de Enfermería de la Universidad de Trujillo, escenario de realización del presente trabajo de investigación, no escapa a la realidad nacional que vive nuestro país. Esta facultad, con un funcionamiento ininterrumpido desde su creación, el 10 de mayo de 1965, reglamentada por la ley universitaria número 17437 de 1960, como parte de la Facultad de Medicina, determinando 48 años ininterrumpidos en la formación de enfermeras, alcanzando 20 años como facultad. Este proceso de cambio, que se ha ido construyendo con el correr de los años, develando fortalezas y debilidades que han permitido ir avanzando en cambios estructurales de infraestructura, académicos en la reestructuración curricular, que después de 13 años de permanencia del currículo actual 
es pertinente su revisión para la actualización necesaria en relación al tiempo y los cambios propios de la época que exigen caminar acordes con los procesos sociales y con la tecnología para la formación de profesionales que estén involucrados con las necesidades locales, nacionales e internacionales, a las que debemos atender como profesionales de Enfermería del siglo XXI, altamente competitivos y pensadores críticos. ${ }^{1}$

Según el renombrado físico peruano Modesto Montoya, sobre los resultados de la tabla mundial de la ciencia emitidos por el grupo Scimago, mientras la mejor universidad chilena posicionada en el ranking, la Universidad de Chile, ha bajado del puesto 384 en 2009 al escaño 412 en el 2012, la universidad brasileña mejor posicionada, la Universidad de Sao Paulo, ascendió en ese mismo lapso de tiempo del $19 \mathrm{al} 11^{\text {avo }}$ puesto. Según Montoya esto se debe a que el estado Brasileño ha cogido el timón y está entrando "en alta mar", en relación con el desarrollo del pensamiento, para formar profesionales competentes. ${ }^{2}$

El pensamiento crítico, como un proceso intelectual valorativo que posibilita que el ser humano discrimine lo esencial de lo no esencial, la consistencia o inconsistencia, la veracidad o no y la validez o no de una idea o acción humana, con el objetivo de emitir juicios de valor con sustentación empírica o teórica. La ciencia, el arte y la sociedad misma requieren hombres y mujeres críticos/as para poder avanzar. En la historia de la filosofía, de las ciencias y de la humanidad, se encuentran ejemplos que concurren a la comprensión de lo dicho. ${ }^{3}$

Los pensadores críticos usan la lógica, validan las primeras impresiones para cerciorarse de que las cosas son como parecen, comprueban tanto la lógica de su pensamiento como la viabilidad de sus soluciones. Distinguen los hechos de las falacias, dedican el tiempo necesario para verificar la información importante y cerciorarse de que es cierta, apoyan opiniones con evidencias. Reconocen lo que es importante para comprender una situación y lo que no lo es. Aplican el concepto de «causas y efecto». Anticipan las respuestas a sus acciones antes de realizarlas. No emiten juicios hasta que conocen todos los hechos necesarios; son conscientes de los peligros que comporta llegar a conclusiones precipitadas. ${ }^{4}$

Por tanto, para fortalecer la educación en el trabajo de la adquisición progresiva de las habilidades del pensamiento crítico por las estudiantes, los docentes de Enfermería deben lograr una correcta integración de los aspectos teóricos con la práctica en la formación profesional, para así estimular un aprendizaje progresivo en el que la contradicción del pensamiento favorezca la solución de problemas reales y evite los potenciales. La vía lógica de obtención de conocimientos es, sin duda, la formación de los educandos en el área práctica, porque 
en ella desarrollarán una mayor independencia cognitiva en su formación intelectual con la guía de los profesores, que serán los responsables de estimular el conocimiento hacia la correcta solución de los problemas.

La teoría y la práctica acompañadas de la investigación nos permiten recrear el conocimiento que apertura nuevas formas de enseñar y aprender en el proceso histórico de cambio que se da permanentemente por el avance inexorable del tiempo. Por ello, repensar permanentemente sobre formas de mejorar los procesos nos ayuda a crecer en el contexto global. El uso intensivo que actualmente se hace de los conocimientos para la creación del desarrollo de cualquier producto o para la prestación de cualquier servicio, como los de salud, ha hecho que cada día que pasa, el ciclo de vida del conocimiento, sea más corto, denominándose a esto proceso de obsolescencia, donde los métodos tradicionales e informales de transferencia de información en general y conocimientos más en concreto, no pueden seguir el rápido ritmo con que se crean y distribuyen los conocimientos, ya que la globalización elimina demoras, permite utilizar y compartir las novedades casi instantáneamente. Por eso, la tendencia es desarrollar competencias principales usando los conocimientos que permitan mejorar el rendimiento. La importancia estriba en el crecimiento sostenido inteligente, el liderazgo, la satisfacción y la excelencia, para asegurar como producto, profesionales calificados. ${ }^{6}$

Una medida necesaria de la educación superior para la formación de los futuros profesionales sugiere la restitución de los Estudios Generales como medio para asegurar la formación integral y favorecer la maduración vocacional de los estudiantes y la práctica sostenida en todo nivel de la investigación; además de aumento de sus presupuestos con la búsqueda de diversos medios de financiamiento. Esto es fundamental, entre otros aspectos, para que nuestros estudiantes lleguen a ser mejores pensadores críticos. ${ }^{7}$

Es lógico pensar y reconocer que el pensamiento crítico del ser humano se va formando en las etapas iniciales de la vida. Mientras más temprano se inicie, se asegura el buen desempeño universitario del estudiante; pero también es posible el inicio de esta forma de pensar críticamente usando métodos adecuados con apertura para generar nuevos aprendizajes en reaprender. La desconstrucción de los procesos que permite desestructurar o descomponer las estructuras conceptuales de un determinado sistema crea la capacidad de generar las condiciones intelectuales para pensar y producir una ruptura epistemológica en el sistema educativo vigente en la universidad y generar una alternativa en los procesos instructivos y transmitidos que hoy lo rigen. ${ }^{4}$ 
Esta operación intelectual se realiza usando sistemas teóricos y métodos de investigación, desde el método clásico, así como con las investigaciones cualitativas. Son estas las que tienen en su base la teoría de las incertidumbres de Morín. Asimismo, en una línea lógica, se orienta a desarrollar los procesos del pensamiento como construcción mental de los conceptos, los juicios y los razonamientos por los alumnos; pero ambas líneas se desarrollan en relación con la aprehensión de los instrumentos de conocimiento o de los saberes fundamentales de las ciencias, tecnologías y humanidades. Esto significa el dominio de los sistemas teóricos de cada disciplina por parte de los estudiantes, según sea su especialidad o nivel de educación en el cual van a laborar. ${ }^{8}$

La presente investigación se realizó con la finalidad de poder identificar el desarrollo del pensamiento crítico de la estudiante de Enfermería, así como determinar cuáles son los obstáculos que se presentan y qué procesos se van desarrollando. Ello se van dando en su proceso de aprendizaje, se visualiza cómo va mejorando el producto final, que es la estudiante egresada de la facultad de Enfermería.

\section{PROBLEMA}

¿Se desarrolla el pensamiento crítico en la estudiante de la Facultad de Enfermería de la Universidad Nacional de Trujillo?

\section{OBJETIVOS}

a) Identificar el desarrollo del pensamiento crítico en la estudiante de la Facultad de Enfermería de la Universidad Nacional de Trujillo.

b) Analizar el desarrollo del pensamiento crítico en la estudiante de la Facultad de Enfermería de la Universidad Nacional de Trujillo.

c) Categorizar el desarrollo del pensamiento crítico en la estudiante de la Facultad de Enfermería de la Universidad Nacional de Trujillo.

\section{MATERIAL Y MÉTODO}

\section{ESCENARIOS DE ESTUDIO Y CRITERIOS DE SELECCIÓN}

El escenario de estudio estuvo constituido por la Facultad de Enfermería de la Universidad Nacional de Trujillo.

\section{SUJETOS DE INVESTIGACIÓN}

La unidad de análisis de la presente investigación estuvo constituida por 
las nueve estudiantes de la Facultad de Enfermería de la Universidad Nacional de Trujillo: cuatro de ellas de quinto ciclo y cinco de sétimo, que aceptaron participar en forma voluntaria en la presente investigación.

\section{MÉTODO Y TÉCNICAS}

a) Tipo de investigación: cualitativa.

b) Método: dialéctico.

La dialéctica busca una explicación de las contradicciones y conflictos en la sociedad, formulando de esta manera una relación respecto al objeto. El procedimiento refleja el análisis y los resultados elaborados según los principios de la lógica metafísica, que entrelazan de manera ecléctica e inconexa diferentes tendencias pedagógicas. Estas tendencias, que constituyen un reflejo de la praxis educativa, sustentada por propuestas filosóficas; por lo tanto, la aplicación del método dialéctico no depende solo del conocimiento técnico, sino de un apostura intelectual y de una visión social de la realidad (De Souza, 2009).

\section{Control DE CALIDAD DE DATOS}

Determinados por la recolección de datos con los criterios establecidos de rigurosidad.

Credibilidad. El investigador ha captado el significado completo y profundo de las experiencias de los participantes..$^{10}$

Triangulación. La producción disciplinar produce interfertilización de saberes, que en proceso de producción del conocimiento, rompe barreras epistemológicas, teóricas y prácticas. ${ }^{9}$

Originalidad. Se mide por la contribución nueva de una investigación. ${ }^{9}$

Objetivación. Resultado de la interacción entre teoría, método y creatividad del investigador frente al objeto de estudio. ${ }^{9}$

Transfiabilidad. Denominada también traslado, pautas generales para determinar el problema estudiado, con la posibilidad de aplicar ciertas soluciones en otras situaciones similares pero en diferente ambiente..$^{10}$

Coherencia. Propiedad de un discurso lógicamente construido, tanto en el sentido teórico como en el desdoblamiento de todas sus etapas de investigación. ${ }^{9}$

\section{RECOLECCIÓN DE INFORMACIÓN}

En la recolección de la información se usó la entrevista semiestructurada y la observación directa, en forma individual, siguiendo el método dialéctico de 
contrastación. El tiempo empleado fue de 20 minutos en promedio; previamente se había coordinado la hora y lugar para llevar a cabo la entrevista, la cual se realizó en forma privada; luego se analizó la información obtenida de cada entrevista, identificando, analizando y categorizando la información recolectada. ${ }^{11}$

\section{PROCESAMIENTO DE DATOS}

Se llevó a cabo simultáneamente con la recolección de la información individual de cada participante; luego de la entrevista, se hizo la transcripción de las entrevistas y se realizó el análisis, determinándose las categorías del estudio. No hay categorías preconcebidas, previas a la investigación. Las categorías se determinaron a partir del análisis realizado de las entrevistas hechas a cada participante; emergieron del estudio de la información que se recogió al hacer el proceso de análisis.

\section{7. ÉTICA DE LA INVESTIGACIÓN}

Se tuvo en cuenta los principios éticos de:

Anonimato, el mismo que ocurre cuando cualquier investigador no puede relacionar al sujeto con los datos.

Libre participación. Se dio por la no manipulación de la información por los intereses personales a favor de la investigación, sino que fue de mutuo acuerdo.

Libre decisión en la participación en la presente investigación.

Honestidad, considerada un valor crucial en el mundo actual, pues la corrupción ha invadido las esferas sociales y administrativas. Honesto es el que hace lo que dice al servicio de la sociedad. ${ }^{3}$

\section{RESULTADOS}

\section{Estudiante 1}

Se desarrolla: como pasan los ciclos.

Al inicio no tenemos: está para desarrollarlo.

Se va desarrollando: exigiéndonos más.

Creo que no todas las alumnas: llegamos a tenerlo.

Tenemos que ir desarrollándolo: poco a poco.

Categoría Primaria:

Dicotómica 


\section{Se desarrolla}

1. Como pasan los ciclos.

\section{No se desarrolla}

1. Al inicio no tenemos está para desarrollarlo.

2. Creo que no todas llegamos a tenerlo.

\section{En proceso}

1. Se va desarrollando exigiéndonos más.

2. Tenemos que ir desarrollándolo poco a poco.

\section{Estudiante 2}

Para el cuidado del paciente es muy importante.

Trabajamos con seres humanos; no podemos equivocarnos.

Debemos prepararnos con bibliografía.

Cada proceso que vamos a hacer debe tener base científica; desarrollamos una buena atención.

Tenemos que especializarnos.

Categoría primaria:

Dicotómica.

\section{Se desarrolla}

1. Cada proceso que vamos a hacer: debe tener base científica, desarrollamos una buena atención.

\section{En proceso}

1. Debemos prepararnos con bibliografía.

\section{Categoría secundaria:}

1. Para el cuidado del paciente es muy importante.

2. Trabajamos con seres humanos no podemos equivocarnos.

3. Tenemos que especializarnos.

\section{DISCUSIÓN}

Los resultados de la presente investigación se exponen en lo que ilustra el proceso, por medio del cual se llegó a las estructuras particulares de los casos estudiados y a la estructura general, que los integran.

De los discursos se pudo desprender elementos que identifican el desarrollo del pensamiento crítico en la estudiante de la Facultad de Enfermería de la Universidad de Trujillo, según su valoración individual.

Considerando la categorización de la información obtenida como primaria dicotómica, cuando considera que se da el desarrollo del pensamiento crítico en 
su formación académica; en contradicción, considera que no se da el desarrollo del pensamiento crítico y en paralelo identifica que hay un desarrollo del pensamiento crítico en proceso.

Por otro lado, hay una categorización secundaria que considera otros aspectos en el desarrollo del pensamiento crítico, que están presentes y se identifican en los discursos.

La exigencia que necesita la estudiante para el desarrollo del pensamiento crítico parte de su exigencia personal en desarrollar diversos procesos, métodos, estrategias que favorezcan su aprendizaje; pero, a la vez, el docente universitario debe convertirse en un propulsor de estrategias metodológicas dinámicas, ya que estamos ante una generación de estudiantes expertos en el manejo del internet, interactivos, multimedia que hacen varias actividades casi simultaneas con gran agilidad mental. De esto se desprende que el docente debe ser un facilitador que logra que el estudiante hable, escriba, discuta en grupo, trabaje con la mayor autonomía y el docente monitoree en forma individual. Ello requiere cambios de paradigma de los esquemas tradicionales a nuevos que se construyan junto con el estudiante. ${ }^{12}$

Esto exige capacitación del docente en el uso de técnicas para mejorar el proceso de aprendizaje de sus estudiantes; enseñar qué estudiar bien implica usar técnicas que permitan rentabilizar de forma efectiva las horas de trabajo, las técnicas de síntesis, las más usadas que engloban el resumen y el esquema, las mismas que se usan conjuntamente o en forma independiente, con el fin de extraer los contenidos importantes y afianzar los conocimientos. ${ }^{13}$

Para el desarrollo del pensamiento crítico los espacios favorables para la generación de nuevas ideas creativas en los estudiantes son: la lluvia de ideas, trabajos colaborativos interdisciplinarios, experimentación, improvisación, meditación, prototipos, lectura de temas sobre innovación. Por ello, conviene estimular a los alumnos universitarios para que sientan que el pensamiento original es valorado y alentado, rompiendo la rigidez del pensamiento rutinario y convencional. Así, las universidades pueden dejar de ser institutos técnicos y convertirse en escenarios de exploración, experimentación, razonamiento libre y producción de ideas originales. ${ }^{14}$

Los buenos docentes, que siendo distintos, pueden ser muy valiosos para la vida de los estudiantes, independientemente de la personalidad y el estilo pedagógico, es necesario que dominen sus temas, que sean personas honestas, no dogmáticas, con mente libre y abierta. No existe un buen maestro a secas, en el vacío, que sea bueno para cualquier contexto y cultura institucional, porque 
el impacto del docente se siente a través del vínculo que establece con cada uno de sus alumnos y grupos, lo cual es distinto en cada contexto. Se observa cómo es que logra que sus alumnos se motiven y aprendan. ${ }^{15}$

El docente como mediador en el proceso enseñanza aprendizaje, propicia el debate permanente y no es el sabio que tiene todas las preguntas y respuestas, siendo los estudiantes los creadores de las respuestas. Sin duda, un enfoque de este tipo permitiría que los alumnos exploren las infinitas aristas de su interés y curiosidad que en conjunto, escuchándose entre dos los estudiantes tendrían un aprendizaje mucho más relevante, profundo y la formulación de implicancias para sus vidas en estos tiempos, que difícilmente se lograría por la vía convencional de preguntas del profesor o examen, y respuestas directas del alumno, generalmente mecánicas, memorizadas, estereotipadas o de repetición de lo que dice el profesor. La sugerencia es intentar; así las capacidades de los estudiantes nos sorprenderán gratamente. ${ }^{16}$

\section{CONCLUSIONES}

Las conclusiones finales dieron dos categorías: primaria y secundaria. En la categoría primaria, dicotómica, se desarrolla el pensamiento crítico poco a poco con apoyo de las docentes; no se desarrolla por falta de motivación, confianza, temor, miedo y en proceso de desarrollo exigiéndonos más. La categoría secundaria es importante para el cuidado del ser humano.

La propuesta radica en determinar currículos, sílabos actualizados permanentemente, de acuerdo a la tecnología y metodología actual en los procesos de enseñanza aprendizaje activos, que propicien el desarrollo del pensamiento crítico.

\section{REFERENCIAS BIBLIOGRAFICAS}

1 N. Cantuarias; I. Moreira; M. Rubio. Inserción de la Enfermería en la Administración Académica Universitaria. Luchas, conquistas y desafíos. Perú. 2005.

2 J. PÉREZ JiMÉNEZ. La Educación en el fondo de la tabla. Diario La Industria. Trujillo-Perú. 14 de abril del 2013.

3 A. S. Moya Obeso. Pensar/Investigar en la era del conocimiento. Lima - Perú. 2010

4 J. Ibarra, F. Conadas.; M. J. Vílchez. Desarrollo del pensamiento crítico en Enfermería. España. 2009.

5 J. DeRrida, N. LOPEZ. La de-construcción curricular. Colección Seminarium Magisterio. Bogotá-Colombia. 2001.

6 A. Del Moral Bueno, E. Rodríguez Fernández., A. Rodríguez Paton, S. Suarez, J. Pazos, JuAn. Gestión del Conocimiento. Madrid-España. 2007. 
7 S. LeRner Febres. Universidad Crisis y Alternativas. Diario la Republica. Lima-Perú. 27 de Febrero del 2011.

8 E. MORÍn. Educar en la era planetaria. Barcelona-España. 2003.

9 M. C. De Souza Minayo. La Artesanía de la Investigación Cualitativa.Buenos Aires-Argentina. 2009.

10 R, Hernandez Sampieri, C. Fernandez Collado, P. Baptista Lucio. Metodología de la Investigación. México D.F. 2010.

11 M. MARTínEZ. Cómo hacer un buen proyecto de tesis con un modelo Cualitativo. Perú. 2009.

12 LeON Trahtemberg. Debate de una ley retrógrada. Diario La Industria, de Trujillo-Perú. 19 de febrero 2012.

13 M. VÁZqUez ReINA. Técnicas para mejorar proceso de aprendizaje. Diario La Industria. Trujillo Perú. 19 de febrero 2012.

14 León Trahtemberg. Los buenos docentes. Diario la Industria, de Trujillo. 13 de marzo 2011.

15 LEÓN TRAHTEMBERG. Reconocer a un buen profesor. Diario La Industria, de Trujillo-Perú. 01 de setiembre del 2013.

16 León Trahtemberg. El provechoso hábito de la lectura. Diario la Industria, de Trujillo-Perú. 5 de febrero del 2012.

Dirección: Mz. H Lote 20 Urb. Los Cedros de Trujillo.

Correo electrónico: delly60@hotmail.com 